\title{
PROGRAMAS DOCTORALES EN CHILE: UNA PROPUESTA TIPOLÓGICA PARA INTERPRETAR SU DIVERSIDAD ${ }^{1}$
}

\author{
Pablo Baeza ${ }^{2}$
}

\begin{abstract}
RESUMEN
Chile ha experimentado un desarrollo acelerado de programas doctorales, tanto cuantitativo -en los últimos años la oferta se duplicó- como cualitativo -creación de programas en nuevas áreas del conocimiento, en contextos territoriales diversos, de carácter interdisciplinar, organizados de forma colaborativa entre universidades-. El estudio busca describir y comprender la diversidad de esta oferta con el objetivo de enriquecer las iniciativas de mejora de la calidad de los programas. Mediante un análisis de conglomerados se construyó una tipología de programas de doctorado basada en nueve variables que dan cuenta de cuatro dimensiones: recursos, procesos, productos y resultados. La tipología describe y transparenta la diversidad horizontal dentro del sistema, diferenciándose de la lógica de otras herramientas, como los rankings. Los resultados muestran una oferta organizada en torno a cinco tipos de programas: fuertes, consolidados, emergentes, en desarrollo y débiles. La diversidad del sistema tiene un carácter ambivalente: de un lado, desarrollo de programas en nuevas áreas del conocimiento, en diversos contextos territoriales y con nuevas características; de otro, las grandes diferencias entre los tipos de programas muestran que hoy el sistema no asegura niveles de calidad equivalentes para los estudiantes de doctorado.
\end{abstract}

Conceptos clave: análisis de conglomerados, calidad, diversidad, programas de doctorado, tipología.

\section{DOCTORAL PROGRAMS IN CHILE: A TYPOLOGICAL PROPOSAL TO EXPLORE THEIR DIVERSITY}

\section{ABSTRACT}

Chile has experienced an accelerated development of doctoral programs. In recent years, the offer has doubled, and the variety of options has significantly expanded with the creation of programs in new areas of knowledge, in different territorial contexts, of an interdisciplinary nature, organized collaboratively among universities. The study seeks to describe and understand the diversity of this offer with the aim of enriching quality improvement initiatives for the programs. Through a cluster analysis, a typology of doctoral programs was constructed based on nine variables that account for four dimensions: inputs, processes, outputs and outcomes.

1 Agradezco los valiosos comentarios de Ricardo Reich y Christian Blanco en la fase de preparación de este trabajo. El resultado final es de mi total responsabilidad.

2 Comisión Nacional de Acreditación, Santiago, Chile. Contacto: pbaeza@cnachile.cl 
The typology describes and reveals the horizontal diversity within the system, differing from the logic of other tools, such as rankings. The results show an offer organized around five types of programs: strong, consolidated, emerging, developing and weak. The diversity of the system has an ambivalent character: on the one hand, the development of programs in new areas of knowledge, in different territorial contexts and with new characteristics; on the other, the vast differences between the types of programs show that the system does not currently guarantee equivalent quality levels for doctoral students.

Keyconcepts: cluster analysis, diversity, doctoral programs, quality, typology.

\section{Introducción}

En cinco décadas de desarrollo de los programas de doctorado en Chile, el panorama ha cambiado radicalmente. La oferta aumentó desde poco más de 20 programas a principios de la década de 1990 a cerca de 270 en 2016. La matrícula se incrementó desde no más de 100 estudiantes a principios de los ochenta a más de 5.500 en 2016 (Servicio de Información de Educación Superior, SIES, 2015; 2016a). A su vez, los graduados pasaron de 16 en 1985 (Brunner, 1986) a 685 en 2015 (SIES, 2016b). Desde el punto de vista de las instituciones, en las dos primeras etapas de desarrollo, entre 1968-1998 (Devés y Marshall, 2008), solo las universidades más consolidadas dentro del Consejo de Rectores de las Universidades Chilenas $^{3}$ (CRUCH) implementaron programas doctorales. Al día de hoy, como resultado de procesos de diferenciación y diversificación, más de 35 universidades, estatales y privadas, desarrollan programas de doctorado en un amplio espectro de áreas del conocimiento (Baeza, 2017).

Esta investigación busca comprender la diversidad en la oferta de doctorado en Chile, con el objetivo de enriquecer los elementos de análisis para el diseño de políticas de desarrollo y aseguramiento de la calidad de los mismos. A partir de un análisis de conglomerados se construyó una tipología de los programas, que da cuenta de las disparidades y similitudes en términos de cuatro dimensiones:

3 El CRUCH agrupa a las 18 universidades estatales de Chile y nueve universidades privadas tradicionales. 
recursos, procesos, productos y resultados. Las principales preguntas que guiaron la investigación fueron: ¿qué nivel de diversidad existe en la oferta de programas doctorales en Chile?, ¿qué características permiten entender esta diversidad?, a partir de estas características ¿es posible identificar tipos de programas?

El presente trabajo se enmarca en la línea de investigación de la diversidad en la educación superior, la que fundamentalmente se ha desarrollado en el nivel institucional, con análisis de la diversidad dentro de los sistemas de educación superior y análisis comparativos entre sistemas. El aporte principal de este trabajo es que aplica esta línea de investigación al nivel de los programas de doctorado y ofrece una herramienta analítica que permite interpretar, contextualizar y distinguir la oferta doctoral en Chile (en un contexto de implementación de la nueva Ley de Educación Superior, que consagra la obligatoriedad de acreditación de los programas de doctorado, Ley $\mathrm{N}^{\circ} 20.091,2018$ ).

El artículo se organiza en cuatro secciones. En la primera se desarrolla el concepto de diversidad en educación superior y se revisan las herramientas utilizadas para su interpretación (rankings y clasificaciones). La segunda sección describe la estrategia metodológica del estudio y las fuentes de datos. La tercera se divide en dos apartados: el primero presenta los resultados del análisis de conglomerados; el segundo interpreta estos resultados, proponiendo una tipología de programas de doctorado en Chile. La última sección muestra los aportes y conclusiones del estudio.

\section{Calidad y diversidad en educación superior}

La problemática acerca de la diversidad surge en un contexto de cambios en los sistemas de educación superior, que pasan de sistemas con altos grados de regulación y pensados para satisfacer una demanda limitada (y elitista) de estudiantes, a sistemas desregulados, con una fuerte expansión de la oferta (pública y privada) y masificación de los estudiantes (de características heterogéneas).

El tránsito desde una educación de élite hacia una de masas (Trow, 2007) se produce como resultado de una serie de reformas 
en los sistemas de educación superior en la línea de desregular y descentralizar la gestión, fomentar la iniciativa privada en la provisión, establecer la competencia como criterio de distribución de recursos y orientar los estudios superiores hacia el mercado (Didriksson, 2012, Rhoades \& Slaughter, 1997; 2004; Slaughter \& Leslie, 2001). Estas reformas generaron sistemas diversificados, con nuevos modelos de financiamiento, altamente masificados y con un creciente problema de calidad en su oferta (Altbach, 2013; Altbach, Reisberg \& Rumbley, 2009).

Es en este contexto donde comienza el desarrollo de mecanismos de rendición de cuentas y medición de indicadores de gestión con énfasis en la eficiencia de las instituciones de educación superior (Goedegebuure \& Meek, 1997; Harvey \& Green, 1993; Kells, 1992; Martin \& Stella, 2007), pensados como estrategias que conforman una nueva manera de regulación de los sistemas. Asimismo, la calidad de la educación también se convierte en un objeto de evaluación, con determinadas rutinas, estructuras y actividades organizacionales asociadas a su provisión (Harvey \& Green, 1993; Stensaker, 2007).

La reflexión en torno a la diversidad problematiza las nuevas exigencias de los sistemas de educación superior en contextos de demanda masificada, inéditos y dinámicos requerimientos del mercado de trabajo, aparición de incipientes proyectos educativos, entre otras características, en un equilibrio constante con la demanda de asegurar calidad en la educación ofrecida.

\subsection{Interpretando la diversidad: rankings y clasificaciones}

El concepto de diversidad, originalmente desarrollado en el campo de la biología y adaptado para el análisis en educación superior, se concibe como la variedad de entidades dentro de un sistema en un momento de tiempo específico (Codling \& Meek, 2006; Huisman, 1995; 2000; Huisman, Meek \& Wood, 2007; van Vught, 2007; 2008). En la literatura se reconocen varias características positivas de los sistemas de educación superior que cuentan con diversidad: mayores posibilidades de acceso y desarrollo a un abanico más amplio de estudiantes (Birnbaum, 1983; Huisman, 1995; Meek, Goedegebuure 
192 PROGRAMAS DOCTORALES EN CHILE: UNA PROPUESTA TIPOLÓGICA PARA INTERPRETAR SU DIVERSIDAD - P. Baeza

\& Huisman, 2000; Trow, 2000; van Vught, 2007; 2008); creciente especialización y flexibilidad de la oferta vinculada a las nuevas necesidades de las sociedades posindustriales (Altbach et al., 2009; Meek et al., 2000; Rama, 2015; Scott, 2015, van Vught, 2007; 2008); ofrece condiciones para que grupos de interés diferenciados —académicos, políticos, culturales, religiosos — tengan un lugar en el sistema (Altbach, 2013; Meek et al., 2000; van Vught, 2007; 2008). Otro aspecto positivo que se plantea acerca de la diversidad es que a través de comportamientos imitativos, las instituciones pueden aprender unas de otras e implementar innovaciones con bajos niveles de riesgo asociados (Stirling, 2007; van Vught, 2007; 2008).

La necesidad de introducir transparencia en los sistemas de educación superior podría indicarse como el leit motiv de la investigación acerca de la diversidad. En este campo se han desarrollado dos puntos de vista: uno vertical, que analiza las diferencias entre unidades (instituciones, departamentos y programas) en términos de desempeño, prestigio académico y reputación, desarrollando para ello rankings (Ziegele, 2013); y uno horizontal, dirigido a analizar las diferencias entre unidades sobre la base de perfiles característicos, construyendo para ello clasificaciones (Ziegele, 2013).

La principal función de un ranking es crear una lista u ordenación vertical que muestre claramente quién está arriba y quién está abajo. A pesar de la gran heterogeneidad de los sistemas de educación superior en el mundo, la mayoría de rankings World University Ranking (Times Higher Education), Academic Ranking of World Universities (Shanghai Jiao Tong University), QS World University Ranking (Quacquarelli Symonds), solo por citar los más renombrados- se basan en un limitado número de factores: reputación académica, recursos institucionales, calidad de los académicos y productividad (Pusser \& Marginson, 2013). Varias críticas se han planteado en relación con los rankings: que se insertan dentro de las relaciones de poder entre Estados, instituciones de educación superior y grupos de interés, como una herramienta que confiere prestigio, distribuye recursos, define estratificaciones y jerarquizaciones dentro y entre los sistemas (Pusser $\&$ Marginson, 
2013); que tienen un limitado valor hermenéutico, ya que evalúan instituciones asumiendo que son homogéneas, ocultando su heterogeneidad y valores distintivos (Pusser \& Marginson, 2013; van der Wende, 2008; van Vught et al., 2010a); que contienen sesgos hacia las instituciones de mayor prestigio académico (McCormick, 2013; Ziegele, 2013); y que premian a las instituciones intensivas en investigación, fundamentalmente aquellas que publican en idioma inglés en las áreas de ciencias naturales y medicina (Ziegele, 2013).

Ahora bien, desde un punto de vista horizontal, las clasificaciones son herramientas que buscan describir y visualizar la diversidad de las unidades dentro de un sistema (van Vught et al., 2005; 2010a). Esto es, intentan transparentar la diversidad horizontal, identificando diferencias y similitudes de las unidades y agrupándolas en conjuntos homogéneos (van der Wende, 2008; Ziegele, 2013). Se pueden distinguir dos tipos de clasificaciones (Ziegele, 2013): de mapeo y tipologías. Las del primer tipo son descriptivas y buscan mostrar qué es lo que están haciendo las instituciones, sin construir un orden rígido y predeterminado sino sujeto a diferentes características y necesidades de los propios usuarios (estudiantes, académicos, autoridades y administradores, entre otros). La clasificación de las instituciones de educación superior europeas, U-Map, desarrollada por el Center for Higher Education Policy Studies (van Vught et al., 2010a), es un buen ejemplo de este tipo de herramienta de perfilamiento institucional.

El segundo subtipo de clasificaciones es la tipología, que combina elementos empíricos y descripciones acerca del tipo de unidades (Ziegele, 2013). Hay tipologías basadas en técnicas idiográficas y en técnicas nomotéticas (Bailey, 1994). Las técnicas idiográficas se basan en criterios conceptuales a priori para construir categorías; las técnicas nomotéticas desarrollan estrategias empíricas para agrupar las unidades en categorías (Bailey, 1994; McCormick, 2013). Dentro de las primeras el ejemplo paradigmático es la Carnegie Classification (McCormick, 2013); dentro de las segundas se pueden señalar los trabajos de Brint, Riddle y Hanneman (2006) y Cheol Shin (2009), entre otros. Bailey (1994) define las técnicas nomotéticas como taxonomías (distinguiendo su carácter empírico frente al 
carácter conceptual de las tipologías), y propone un modelo que combina lo conceptual con lo empírico: tanto si se comienza desde la teoría (nivel idiográfico-conceptual) como si se comienza desde los datos (nivel nomotético-empírico), la combinación de ambos niveles constituye lo que denomina una clasificación operacional (Bailey, 1994).

En el contexto chileno se han desarrollado tanto rankings (construidos por revistas como América Economía y Qué Pasa, o Universitas) como clasificaciones. Ambos tipos de esfuerzos se han orientado hacia el análisis de la diversidad institucional entre universidades. Muñoz y Blanco (2013) señalan que se observan tres etapas en los intentos por clasificar las universidades en Chile. En una primera (histórica) predominan distinciones del sistema basadas en los pares tradicional/privado, estatal/privado, $\mathrm{CRUCH} /$ no $\mathrm{CRUCH}$, que se relacionan con aspectos fundacionales y de pertenencia o no al orden estatal.

La segunda etapa (tipológica) surge como reacción a estas clasificaciones tradicionales e incorpora variables relativas a condiciones de funcionamiento (tamaño, selectividad, oferta, acreditación y composición de la matrícula, entre otras), pasando de categorizaciones binarias a multidimensionales. Dentro de estos esfuerzos, orientados a relativizar y complejizar las clasificaciones tradicionales - señaladas como arbitrarias e incoherentes (Brunner, 2013) — se encuentran los trabajos de Brunner et al. (2005), Brunner (2009), Parada (2010), Torres y Zenteno (2011), Williamson (2012) y Reyes y Rosso (2013). El método utilizado es deductivo: parte de distinciones conceptuales (basadas en la combinación de variables) que se constituyen en tipos (universidades de investigación, de docencia, especializadas, selectivas y regionales, entre otros), los que son descritos a través del uso de datos empíricos (matrícula, puntajes en la Prueba de Selección Universitaria, planta docente, características sociodemográficas estudiantes, acreditación, etc.).

Muñoz y Blanco señalan que la tercera etapa (taxonómica) sería prefigurada por el trabajo de Reyes y Rosso (2013), debido al uso que hacen de los datos empíricos, y comenzaría con su propio trabajo, en 
el que "los factores adscritos a las universidades pierden relevancia frente a otros elementos distintos de los criterios institucionales, dando lugar a clasificaciones de carácter estadístico" (Muñoz y Blanco, 2013, p. 192). Los autores utilizan el análisis factorial (con 15 variables) como un análisis de conglomerados de facto (Bailey, 1994), ya que para construir sus posiciones taxonómicas se centran en los valores de un factor.

Como resultado de su análisis, Muñoz y Blanco proponen una taxonomía de las universidades chilenas de cinco tipos:

- Universidades de investigación: Poseen alto número de programas de doctorado y magíster, proyectos de investigación Fondecyt, publicaciones indexadas, y los índices de selectividad de estudiantes más altos del sistema.

- Universidades de acreditación. Instituciones con buenos resultados de acreditación general y con áreas electivas de acreditación (vinculación con el medio, investigación, docencia de posgrado), con una importante participación en la oferta de posgrado, proyectos Fondecyt y publicaciones indexadas, y de selectividad media.

- Universidades masivas. Agrupan un tercio de la matrícula nueva y total del sistema, con una oferta centrada en el pregrado, con un gran número de sedes a lo largo del país, y con una baja selectividad.

- Universidades elitistas. Su principal característica es su grado de selectividad (media-alta), además de poseer buenos niveles de acreditación, una oferta equilibrada entre pregrado y posgrado.

- Universidades no elitistas. Se trata de instituciones enfocadas en pregrado, con un alto número de sedes, bajo nivel de acreditación, baja concentración de matrícula y bajos índices de selectividad.

El presente trabajo se enmarca dentro de la línea de análisis de la diversidad horizontal a través de la construcción de una clasificación como resultado de la combinación entre una etapa conceptual y una empírica, cercana a lo que Bailey (1994) llama una clasificación operacional. El análisis se inicia desde la teoría paso necesario para establecer las variables sustantivas_-, desarrolla 
un análisis estadístico de conglomerados con el cual se agrupan las unidades por similitud, y se cierra con una síntesis conceptualempírica, que traduce los conglomerados estadísticos en una tipología de programas de doctorado. Esta tipología no busca una clasificación exhaustiva de los mismos en determinados grupos ni jerarquizarlos como un ranking —esfuerzo de tipo estático y cuyos resultados se hacen obsoletos en el tiempo-, sino la construcción de una herramienta que permita interpretar, contextualizar y diferenciar la oferta doctoral.

Para definir las dimensiones y variables a considerar en la construcción tipológica, se utiliza el marco conceptual que considera la educación como un sistema productivo, donde una serie de recursos (inputs) son transformados - por medio de un entramado de procesos (processes) — en un conjunto de productos (outputs), que generan resultados (outcomes) dentro y fuera del sistema (van Vught et al., 2010b). Se optó por este marco conceptual porque considera a los programas como agentes activos de su propio desarrollo, poniendo énfasis no solo en sus productos y resultados, sino también en los recursos que logran captar y los procesos que implementan para desarrollarlos.

En este esquema, los programas deben lograr captar la mayor cantidad y mejor calidad de recursos —investigadores, estudiantes, fondos, becas, etc.- , con el objeto de transformarlos en productos. Esta transformación se logra a través de la implementación de un conjunto de procesos que los programas ponen en marcha de forma consistente con sus objetivos (docencia, investigación, vinculación con el medio): políticas, procedimientos y estructura de gestión, currículo, servicios, apoyo académico a los estudiantes, relación tutorestudiante, entre otros. Estos procesos buscan generar productos inmediatos (graduados, publicaciones o empleabilidad). Por último, en un nivel más estratégico de sus objetivos, los programas buscan satisfacer aquello que declaran en su visión y misión, y que se relaciona con el impacto que pretenden generar en la sociedad desarrollo científico y aplicado, redes de investigación, bienestar en la comunidad, entre otros- (van Vught et al., 2010b). 


\section{Metodología}

Las cuatro dimensiones de análisis seleccionadas — recursos, procesos, productos y resultados- permiten fijar puntos de referencia (benchmarks) entre los programas, agruparlos y caracterizarlos en torno a estos ejes. Para operacionalizar estas dimensiones en variables se consideraron los siguientes criterios generales:

- que las variables tuvieran mediciones para todos los casos (programas doctorales);

- que las mediciones de las variables estuvieran validadas por instituciones del sistema de educación superior;

- que las variables midieran realidades de los programas de forma directa y no de forma indirecta a través de variables institucionales.

\subsection{Recursos}

En un enfoque sistémico, donde los programas reciben recursos y producen resultados hacia el entorno, esta dimensión se relaciona con la pregunta ¿qué entra en el programa? Esto se traduce en el número y particularidades de los estudiantes matriculados, en las características de los académicos asociados a los claustros (experiencia, productividad científica, entre otros), en los recursos financieros que entran al programa (becas, fondos de investigación, entre otros) y en los recursos de infraestructura y equipamiento con los que cuenta el programa (laboratorios, bibliotecas, entre otros).

Teniendo en cuenta la información disponible, esta dimensión se centra en el número de estudiantes (matrícula total y de primer año), sus características (edad promedio, estudiantes extranjeros), y el número de becas de doctorado entregadas por la Comisión Nacional de Ciencia y Tecnología (Conicyt). Las variables matrícula total y matrícula de primer año dan cuenta del principal recurso que debe asegurarse para cada programa: estudiantes. Se incluyó esta última variable para detectar la capacidad de los programas más jóvenes de captar alumnos (por ejemplo, 27 programas iniciaron sus actividades en 2015 y 2016). Contar con un flujo de estudiantes constante les 
permite proyectarse a largo plazo, cuestión clave para el desarrollo y sustentabilidad de sus líneas de investigación.

Las variables edad promedio de los estudiantes, becas de doctorado y proporción de estudiantes extranjeros se incluyen como indicadores de la calidad de los estudiantes que recibe cada programa. Respecto de la edad, existe abundante literatura que desarrolla la relación entre carrera científica, edad y posición de los investigadores. Merton y Zuckerman (1973) plantearon diferencias en el foco entre investigadores jóvenes (más propensos a encontrar nuevos hallazgos y menos basados en resultados anteriores) y adultos (quienes ejercen una continuidad disciplinar vinculando su trabajo con lo hecho anteriormente en el campo); se ha evidenciado una relación entre los ritmos de publicación y la edad, con una curva en forma de "U" invertida, donde el promedio de publicaciones se incrementa con la edad y alcanza su máximo a un cierto punto de la carrera (usualmente entre los 50 y 59), donde empieza a declinar — dinámica que también es influida por las posiciones académicas por las que pasa un investigador a lo largo de su carrera (Aksnes, Rørstad, Piro \& Sivertsen, 2011; Barjak, 2006; Diem \& Wolter, 2013; Gingras, Larivière, Macaluso \& Robitaille, 2008; Rørstad \& Aksnes, 2015)_; otra investigación reveló que la edad tiene influencia en la probabilidad de trabajar como investigador, en el sentido de que a menor edad más oportunidad tiene un científico de trabajar como tal (Boosten, Vandevelde, Derycke, te Kaat \& van Rossem, 2014).

En suma, si bien la relación entre edad y carrera científica no es lineal y está atravesada por otros factores relevantes - posición académica (Diem \& Wolter, 2013; Rørstad \& Aksnes, 2015), género (Aksnes et al., 2011), disciplina (Sabharwal, 2013), efecto acumulativo o efecto Mateo (Merton, 1968)_, en esta investigación se asume que mientras menor sea el promedio de edad de los matriculados en los programas doctorales mayor será su orientación y vinculación con actividades de investigación, por lo que contribuirán en mayor medida a la generación de productos del mismo programa; por el contrario, mientras mayor sea la edad de los matriculados, se asume que los estudiantes utilizan en mayor medida los programas de doctorado con fines profesionales y menos ligados a la investigación. 
Respecto de las becas, debido a que estas se otorgan como resultado de un proceso de evaluación de expertos, aquellos programas que reciben más estudiantes becados no solo aseguran recursos económicos para el ciclo de estudio, sino además aquellos mejor evaluados desde el punto de vista de su registro académico y potencial investigador. En relación con la proporción de estudiantes extranjeros, muestra la capacidad de los programas por captar nuevos estudiantes, que tienen el potencial de generar redes internacionales de colaboración.

\subsection{Procesos}

Esta dimensión responde a la pregunta ¿qué se hace con los recursos que entran al programa?, y se relaciona con los programas de estudio, los servicios ofrecidos a los estudiantes, el desempeño disciplinar de los académicos, el apoyo académico entregado a los estudiantes, las políticas, procedimientos y estructura de gestión de los programas, entre otros. Algunos de estos elementos se encuentran a disposición del público general, fundamentalmente en las páginas web de los programas, pero escasamente sistematizados y validados. Otros aspectos son incorporados en los procesos de acreditación que realiza CNA (Comisión Nacional de Acreditación, CNA, 2016), pero no son informados públicamente por parte de los programas. Sabiendo que esta dimensión tiene un componente de evaluación predominantemente cualitativo (basado en el análisis experto de pares), no se encuentran en el sistema indicadores directos de evaluación (cuantitativos y/o cualitativos) que informen acerca de ella: por ejemplo, si bien existe un repositorio de publicaciones científicas de Conicyt para cada investigador, no hay un registro público y actualizado de la composición de los claustros de los programas ofertados en el sistema, por lo que la tarea de vincular cada investigador con cada programa es compleja y excede con mucho el objetivo de esta investigación.

Debido a estos déficits de información, se incorporaron dos variables que dan cuenta de forma indirecta de los procesos que los programas ponen en marcha en sus actividades de enseñanza. El año de inicio de actividades indica la experiencia adquirida por los 
programas. Si bien no necesariamente los más antiguos son los de mayor calidad, la evidencia muestra que los que iniciaron de forma más temprana sus actividades se implementaron en las universidades más consolidadas, con grupos de investigadores afianzados y, en general, poseen mejores evaluaciones de calidad (Baeza, 2017; Munita \& Reyes, 2012).

Asimismo, dado que se ha mostrado un impacto positivo de los procesos de acreditación en la gestión y desempeño de los programas de estudio (Celis \& Véliz, 2017; Centro Interuniversitario de Desarrollo, Cinda, 2012; Dooner et al., 2016; Zapata \& Tejeda, 2009), el número de procesos de acreditación realizados es un indicador que permite valorar los programas que han trabajado de forma continuada en los seis criterios que contempla CNA, y distinguirlos de aquellos que no se han sometido a procesos de acreditación, o bien, poseen poca experiencia (uno o dos procesos).

\subsection{Productos}

La tercera dimensión se relaciona con la pregunta ¿cuántos productos genera el programa? Los productos tienen que ver con los graduados; número de publicaciones producidas por estudiantes, egresados y académicos; redes de investigación generadas por el programa, entre otros. En esta dimensión también nos encontramos con una realidad dispar, ya que si bien se tiene acceso al número de graduados, no se encuentran disponibles los datos de la progresión académica de cada programa (retención, tasas de graduación), ni la productividad científica de los claustros, debido al vacío que se mencionó en el punto anterior. Esta información sí considera en los procesos de acreditación de CNA, pero es un dato entregado por cada programa al momento en que entra a proceso, con lo que surge el consiguiente problema temporal de comparación entre ellos. Por estas razones se incluye la variable graduados como el único indicador fiable y comparable para todos los programas de esta dimensión.

\subsection{Resultados}

Esta dimensión busca responder a la pregunta ¿cuál es el impacto del programa?, y es posiblemente la más débil desde el punto de vista 
de la información disponible para su evaluación. No existe evidencia de los aprendizajes o capacidades adquiridas por los graduados de doctorado; de la contribución de la investigación generada por el programa a la disciplina en la que se enmarca; de la contribución del programa al desarrollo académico y científico de la universidadciudad-país; de su impacto en otros programas de doctorado o posgrado, entre otros. Muchos de estos aspectos —al igual que los de la dimensión procesos- son evaluados dentro de la acreditación de CNA y no están disponibles para un análisis de tipo estadístico (con datos cuantitativos y cualitativos).

Es por esto que se incorpora la variable acreditación del programa como un indicador global de calidad del mismo y de la valoración (por parte de expertos del área) de su impacto. Se asume que la obtención de más años de acreditación implica que un programa doctoral ha alcanzado un mayor impacto, tanto en el campo de investigación en el que se desarrolla (a través de su producción científica de académicos, estudiantes y egresados), como en relación con el desarrollo de su entorno. La Tabla 1 resume las nueve variables incorporadas en el análisis de conglomerados.

La unidad de análisis son los programas de doctorado que se desarrollan en universidades chilenas que figuraron en la oferta académica de 2016, esto es la oferta reconocida por el Ministerio de Educación de Chile, Mineduc. El total de programas incorporados asciende a 266. Los programas conjuntos (11) se consideraron como independientes unos de otros en el análisis, ya que cada cual posee diferentes niveles de matrícula, graduados, becas, entre otros elementos. 


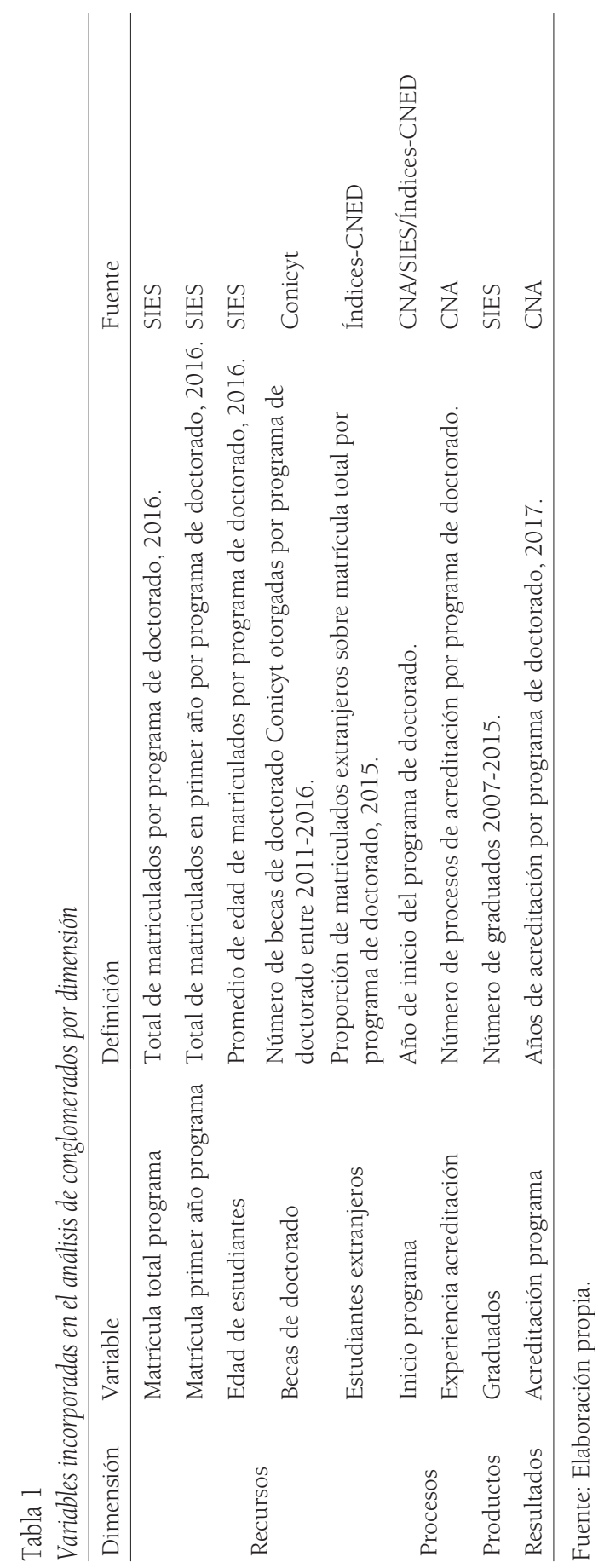




\subsection{Análisis de conglomerados}

El análisis de conglomerados o cluster analysis es una técnica multivariable de clasificación. Con ella se busca agrupar una serie de unidades en conjuntos (conglomerados o clúster). El objetivo es que las unidades clasificadas en cada conglomerado sean lo más similares entre sí y diferentes respecto de las unidades clasificadas en los otros conglomerados (Bailey, 1994; Cea D'Ancona, 2004). Lo que define a un conglomerado es su cohesión interna (homogeneidad) y su aislamiento externo (separación), permitiendo descubrir estructuras latentes en los datos (Everitt, Landau, Leese \& Stahl, 2011). El objetivo del análisis es conseguir la solución más parsimoniosa, esto es, la estructura de datos más simple que represente grupos homogéneos (Cea D’Ancona, 2004). Para esto se deben tomar decisiones respecto de las variables a utilizar, de la estandarización de las métricas de las variables, de la selección de las medidas de distancia y similaridad de las unidades, de la selección del o los métodos de aglomeración que se aplicarán, de la determinación del número de conglomerados y, finalmente, de la interpretación de los conglomerados (Bailey, 1994; Cea D’Ancona, 2004; Huberty, Jordan $\&$ Brandt, 2005; Milligan \& Cooper, 1987).

En el análisis de conglomerados existen dos métodos comúnmente utilizados: los jerárquicos y los no jerárquicos o de partición. Los métodos jerárquicos consisten en la clasificación de las unidades en una serie de particiones secuenciales y pueden operar según dos procedimientos: uno de tipo divisivo, que consiste en la partición sucesiva del conglomerado inicial, que contiene todas las unidades, hasta que existen tantos conglomerados como unidades a clasificar (Everitt et al., 2011); y otro de tipo aglomerativo, el más utilizado, que opera de forma inversa, esto es, su punto de partida son las unidades singulares, las que comienzan a fusionarse de forma iterativa hasta conformar un único conglomerado (Cea D'Ancona, 2004; Everitt et al., 2011).

Los métodos no jerárquicos son denominados también como de partición, porque en ellos se produce solo una partición de datos (Milligan \& Cooper, 1987), al contrario del jerárquico, en el que 
los pasos secuenciales - aglomerativos o divisivos - se encuentran anidados entre sí. La característica de los métodos no jerárquicos es que comienzan a partir de una división inicial de los datos, es decir, se debe especificar inicialmente un número determinado de conglomerados, de acuerdo con un criterio de investigación (teórico o bien a partir del resultado previo de un análisis jerárquico). El procedimiento opera asignando las unidades a los conglomerados a cuyo centro (centroide, que es el valor medio de las variables del conglomerado) se encuentran más próximos (Cea D’Ancona, 2004; Everitt et al., 2011).

En este estudio se utilizó una estrategia combinada, recomendada para disminuir los inconvenientes del uso de uno u otro método por separado (Cea D’Ancona, 2004; Huberty et al., 2005; Milligan \& Cooper, 1987). Se aplicó un procedimiento jerárquico aglomerativo, usando el algoritmo de Ward, con el que se obtuvo el número de conglomerados, composición y centroides. Posteriormente, se realizó un procedimiento no jerárquico K-means, especificando el número de conglomerados y los valores de sus respectivos centroides que resultaron del primer procedimiento.

Para el desarrollo del análisis de conglomerados se utilizó el programa estadístico Stata, en su versión 14.1. En las Tablas 2 y 3 se muestran los estadísticos descriptivos de las variables utilizadas y la matriz de correlación, respectivamente. Entre las variables incorporadas se presentan tres pares que tienen correlaciones altas: matrícula total y becas de doctorado $(0,873)$, matrícula total y graduados $(0,779)$, y becas de doctorado y graduados $(0,807)$. Se mantienen en el análisis por razones teóricas —debido a que incorporan matices sustantivos en los conglomerados- y estadísticas, ya que las correlaciones no son lo suficientemente elevadas como para decidir eliminar las variables (Huberty et al., 2005). 
Tabla 2

Estadísticos descriptivos variables incorporadas en el análisis de conglomerados

\begin{tabular}{lcccccccc}
\hline \multirow{2}{*}{ Variables } & \multicolumn{7}{c}{ Estadísticos } \\
\cline { 2 - 9 } & $\mathrm{N}$ & Media & D.E. & Mín. & Q1 & Q2 & Q3 & Máx. \\
\hline Matrícula total & 266 & 21,2 & 17,6 & 0 & 8 & 16 & 30 & 118 \\
Matrícula primer año & 266 & 3,9 & 3,4 & 0 & 1 & 3 & 6 & 16 \\
Edad de estudiantes & 266 & 34,1 & 5,1 & 25 & 30,1 & 33,2 & 36,9 & 56 \\
Becas de doctorado & 266 & 15,1 & 17,8 & 0 & 0 & 10 & 24 & 91 \\
Estudiantes extranjeros (\%) & 266 & 0,14 & 0,17 & 0 & 0 & 0,08 & 0,24 & 1 \\
Inicio programa & 266 & 2003 & 10,8 & 1935 & 2000 & 2006 & 2012 & 2016 \\
Experiencia acreditación & 266 & 2,2 & 1,5 & 0 & 1 & 2 & 3 & 5 \\
Graduados & 266 & 14,7 & 19,9 & 0 & 0 & 7 & 22 & 125 \\
Acreditación programa & 266 & 3,3 & 2,8 & 0 & 0 & 3 & 6 & 10 \\
\hline
\end{tabular}

Fuente: Elaboración propia.

Tabla 3

Matriz de correlaciones variables análisis conglomerados

\begin{tabular}{|c|c|c|c|c|c|c|c|c|c|}
\hline Variables & 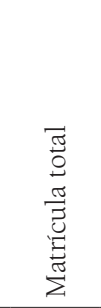 & 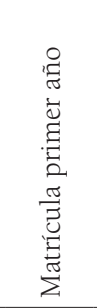 & 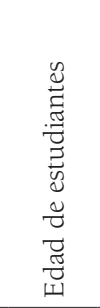 & 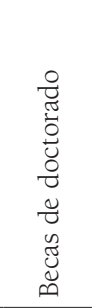 & 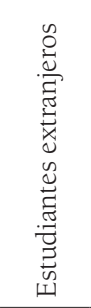 & 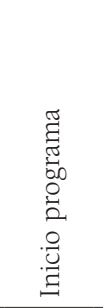 & 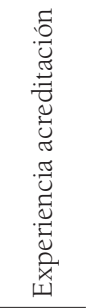 & 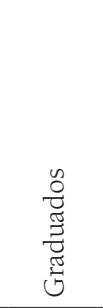 & 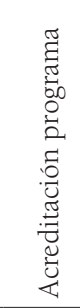 \\
\hline Matrícula total & 1.000 & & & & & & & & \\
\hline $\begin{array}{l}\text { Matrícula primer } \\
\text { año }\end{array}$ & 0,689 & 1.000 & & & & & & & \\
\hline Edad de estudiantes & $s-0,101$ & $-0,127$ & 1.000 & & & & & & \\
\hline Becas de doctorado & 0,873 & 0,592 & $-0,277$ & 1.000 & & & & & \\
\hline $\begin{array}{l}\text { Estudiantes } \\
\text { extranjeros }\end{array}$ & 0,004 & $-0,048$ & $-0,056$ & 0.032 & 1.000 & & & & \\
\hline Inicio programa & $-0,414$ & $-0,201$ & 0,225 & $-0,493$ & $-0,085$ & 1.000 & & & \\
\hline $\begin{array}{l}\text { Experiencia } \\
\text { acreditación }\end{array}$ & 0,503 & 0,338 & $-0,286$ & 0,598 & 0,192 & $-0,537$ & 1.000 & & \\
\hline Graduados & 0,779 & 0,524 & $-0,222$ & 0,807 & 0,003 & $-0,586$ & 0,518 & 1.000 & \\
\hline $\begin{array}{l}\text { Acreditación } \\
\text { programa }\end{array}$ & 0,489 & 0,331 & $-0,443$ & 0,654 & 0,134 & $-0,574$ & 0,674 & 0,564 & 1.000 \\
\hline
\end{tabular}

Fuente: Elaboración propia. 
En este estudio se optó por convertir las mediciones de las variables en puntuaciones estandarizadas $Z$. La estandarización de las variables a puntajes $Z$ se relaciona también con la elección del índice de distancia o similaridad del análisis. En este caso, se decidió utilizar la distancia euclídea al cuadrado (en Stata L2squared), recomendada para los métodos de conglomeración de Ward y K-means (Cea D’Ancona, 2004; Huberty et al., 2005).

Una vez construidos estadísticamente los conglomerados de programas doctorales, se realizó una síntesis conceptual-empírica a través de la cual se tradujeron las agrupaciones en una clasificación tipológica. Para ello se utilizó información adicional vinculada tanto con los programas: región en que se imparten, área OCDE del conocimiento, proyectos del programa de Mejoramiento de la Calidad y la Equidad en la Educación Superior, Mecesup, tramo de acreditación, si fue incluido en el Estudio Evaluativo del Mineduc (2014); como a las instituciones que los albergan: tipo de institución, tramo de acreditación, taxonomía de las universidades de Muñoz y Blanco (2013).

\section{Resultados}

Después del primer procedimiento de conglomeración, basado en el método jerárquico de Ward, fue necesario encontrar el número óptimo de conglomerados. Los índices de Calinski/Harabasz y Duda/ Hart son dos de los mejores procedimientos (stopping rules) para determinarlos (Milligan \& Cooper, 1985). Ambos índices los provee Stata, pero no entregan la solución de partición en conglomerados, sino elementos de juicio que deben ser interpretados ${ }^{4}$. Los resultados de ambos índices sugieren la solución de cinco conglomerados como la más ajustada a los datos.

El análisis del dendrograma de este primer procedimiento de conglomeración jerárquica de Ward (Figura 1), muestra que, en una

4 Valores altos del índice de Calinski/Harabasz indican estructuras de conglomerados y valores bajos muestran estructuras menos definidas. A su vez, la interpretación del índice de Duda/ Hart busca encontrar valores altos de Je(2)/Je(1) que correspondan a valores bajos de pseudo T-squared que tengan valores más altos de pseudo T-squared contiguos (StataCorp, 2015). 
mirada vertical, hay dos grandes grupos, cada uno de los cuales está divido en dos y tres subgrupos, a la izquierda y derecha del dendrograma, respectivamente. De la interpretación de los índices de Calinski/Harabasz y Duda/Hart y el dendrograma, se decidió un número de cinco conglomerados para continuar con la siguiente etapa.

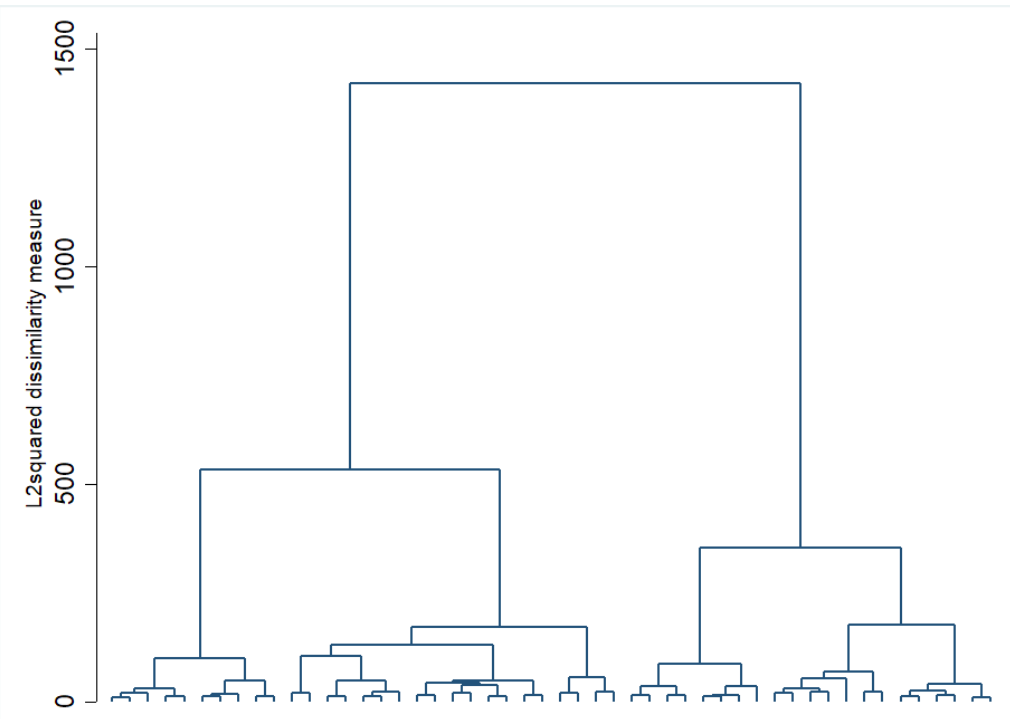

Figura 1. Dendrograma de análisis jerárquico de conglomerados.

Fuente: Elaboración propia.

Se especificó un valor de cinco conglomerados para la aplicación del método de conglomeración no jerárquico iterativo K-means. El análisis de ajuste y validez interna de la conglomeración no jerárquica $\mathrm{K}$-means, se realiza mediante un gráfico de silueta (Figura 2). Esta herramienta gráfica es una ayuda para la interpretación y validación del análisis de conglomerados (Everitt et. al, 2011; Rousseeuw, 1987). En la figura, cada conglomerado está representado por una curva y se muestra la clasificación de las unidades en cada uno de ellos. En el eje de las ordenadas se muestra el valor de la amplitud de la silueta (s), en una escala de - 1 a 1 , para cada unidad (i), mostradas en el eje de las abscisas. Un valor en la silueta igual a 1 corresponde a una clasificación óptima de cada unidad en su correspondiente 
conglomerado, así como un valor negativo indica que esa unidad está más cerca de otro conglomerado.

Se observa que las siluetas de los conglomerados son largas, aunque hay determinadas unidades que no encajan perfectamente en cada uno. Los conglomerados 1, 2, 3 y 5 (de izquierda a derecha en el gráfico) muestran unidades que podrían vincularse con otro. El conglomerado 4 denota un mejor ajuste. El valor medio de la amplitud de la silueta de cada uno de los conglomerados es de: 1 $(0,31), 2(0,36), 3(0,36), 4(0,53)$ y $5(0,21)$.

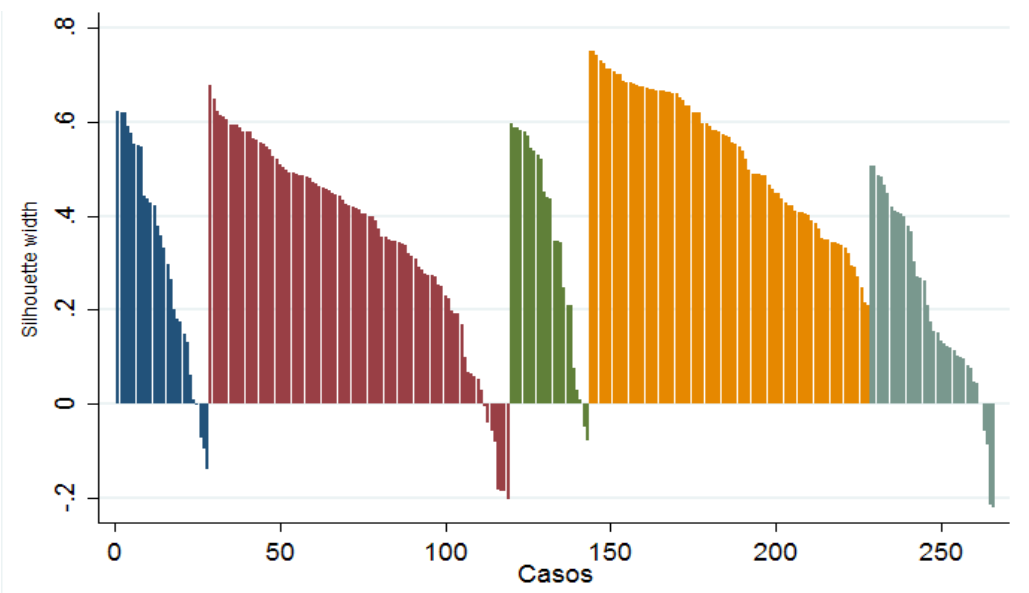

Figura 2. Siluetas de conglomerados método K-means ( $k=5)$.

Fuente: Elaboración propia.

Un procedimiento para validar el análisis de conglomerados es comparar el valor de $(k)$ de diferentes soluciones, donde $k$ corresponde al número de conglomerados (Rousseeuw, 1987). El valor de para la solución de cinco conglomerados corresponde a 0,39 ; las soluciones de 2, 3 y 4 conglomerados obtienen valores de de 0,51,0,45 y 0,40, respectivamente; y las soluciones de 6, 7 y 8 , valores de de 0,38, 0,34 y 0,34, respectivamente. A pesar de que las tres primeras soluciones obtienen valores de superiores, la solución de cinco conglomerados cumple con la triple condición de parsimonia (simplicidad), homogeneidad interna de los grupos (similaridad-separación) y sentido lógico-sustantivo (consistencia teórica). 


\subsection{Interpretación de los conglomerados}

Los resultados de los conglomerados se interpretan en relación con las dimensiones recursos, procesos, productos y resultados que guiaron el análisis. El objetivo es mostrar las características centrales de cada uno de los conglomerados - aquello que los define como grupo y aquello que los diferencia del resto- para realizar una tipología de la oferta de programas de doctorado que permita interpretar la diversidad del sistema. Las siguientes tablas muestran los valores medios de las variables no estandarizadas de cada conglomerado, esto es, los valores de los centroides de cada uno en su unidad de medida original.

\subsubsection{Recursos}

La Tabla 4 muestra las variables de la dimensión recursos. Se observa que los programas de doctorado del conglomerado 1 poseen mayor número de matrícula total y de primer año, duplicando a los del conglomerado 2. Los conglomerados 3, 4 y 5 presentan niveles de matrícula total y de primer año similares, entre 10-14 y 2-3 estudiantes, respectivamente. En relación con las becas de doctorado entregadas por Conicyt en los últimos seis años (2011-2016), el conglomerado 1 recibió la mayor cantidad promedio de becas (cerca de nueve por año), seguido por el conglomerado 2 (cuatro becas por año). Los programas de los conglomerados 3 y 4 recibieron menos de una beca por año. El conglomerado 5 recibió en promedio una beca por año. Si consideramos la relación entre la matrícula de primer año con el promedio de becas anuales conseguidas por cada conglomerado, se obtiene un cálculo aproximado de la cobertura de las becas sobre la matrícula: para los conglomerados 1 y 2 esta sería de $93 \%$ y $85 \%$, respectivamente; un $10 \%$ para los programas del conglomerado 3, y un $24 \%$ y $40 \%$ para los conglomerados 4 y 5 , respectivamente. 
210 PROGRAMAS DOCTORALES EN CHILE: UNA PROPUESTA TIPOLÓGICA PARA INTERPRETAR SU DIVERSIDAD - P. Baeza

Tabla 4

Medias de variables de la dimensión recursos por conglomerado

\begin{tabular}{lrrrrr}
\hline \multirow{2}{*}{ Variables dimensión recursos } & \multicolumn{5}{c}{ Conglomerados } \\
\cline { 2 - 6 } & \multicolumn{1}{c}{2} & \multicolumn{1}{c}{3} & \multicolumn{1}{c}{4} & \multicolumn{1}{c}{5} \\
\hline Matrícula total & 57,6 & 25,7 & 14,4 & 9,7 & 13,4 \\
Matrícula primer año & 9,3 & 4,2 & 2,9 & 2,4 & 2,9 \\
Edad de estudiantes & 31,7 & 32,4 & 45,7 & 33,4 & 34,3 \\
Becas de doctorado & 52,1 & 21,4 & 1,8 & 3,5 & 6,9 \\
Estudiantes extranjeros (\%) & 10 & 17 & 5 & 4 & 42 \\
$\mathrm{~N}$ & 28 & 91 & 24 & 85 & 38 \\
\hline
\end{tabular}

Fuente: Elaboración propia.

Respecto del promedio de edad de los estudiantes, los conglomerados 1, 2 y 4 tienen los valores más bajos, entre 31,7 y 33,4 años. El conglomerado 5 muestra edades promedio de cerca de tres años superiores a las del conglomerado 1. La edad promedio de los estudiantes del conglomerado 3 es de 45,7 años, esto es, 14 años por sobre el valor mostrado del conglomerado 1 y cerca de 12 años por sobre la media de la variable en toda la muestra.

En cuanto a la proporción de estudiantes extranjeros sobre la matrícula total, el conglomerado 5 muestra un valor medio del $42 \%$. Con un $17 \%$ le sigue los programas del conglomerado 2, ligeramente superior a la media de la muestra (14\%). Los conglomerados 1, 3 y 4 tienen proporciones de extranjeros en su matrícula inferiores a la media del sistema: 10\%, 5\% y 4\%, respectivamente.

\subsubsection{Procesos}

Los conglomerados 1 y 2 agrupan los programas más antiguos del sistema, cuyos años de inicio de actividades promedio es en 1993 y 1997, respectivamente (Tabla 5). Los conglomerados 3 , 4 y 5 muestran años de inicio entre 2008 y 2010, muy alejados de los 20 años de experiencia promedio de los conglomerados 1 y 2. Respecto de la experiencia en acreditación, variable medida en número de procesos de acreditación realizados con CNA, se aprecia que los conglomerados 1 y 2 tienen experiencias similares, en torno a tres procesos de acreditación promedio. Por su parte, los conglomerados 4 y 5 poseen entre uno y dos procesos de acreditación, 
y el conglomerado 3 no alcanza a tener un proceso de acreditación promedio.

\section{Tabla 5}

Medias de variables de la dimensión procesos por conglomerado

\begin{tabular}{lccccc}
\hline \multirow{2}{*}{ Variables dimensión procesos } & \multicolumn{5}{c}{ Conglomerados } \\
\cline { 2 - 6 } & 1 & 2 & 3 & 4 & 5 \\
\hline Inicio programa & 1993 & 1996 & 2008 & 2010 & 2009 \\
Experiencia acreditación & 3,3 & 3,5 & 0,7 & 1,1 & 1,7 \\
$\mathrm{~N}$ & 28 & 91 & 24 & 85 & 38 \\
\hline
\end{tabular}

Fuente: Elaboración propia.

\subsubsection{Productos}

La variable graduados, que caracteriza la dimensión productos, muestra la suma total de graduados en nueve años (periodo 20072015). Aquí podemos observar que el conglomerado 1 destaca por sobre los otros, con poco más de seis graduados promedio por año en el periodo considerado (Tabla 6). Los programas del conglomerado 2 poseen 21 graduados en el periodo, lo que supone poco más de dos graduados promedio por año. Los restantes conglomerados presentan entre dos y siete graduados en todo el periodo considerado, esto es, menos de un graduado promedio por año.

Tabla 6

Media de variable de la dimensión productos por conglomerado

\begin{tabular}{lccccc}
\hline \multirow{2}{*}{ Variable dimensión productos } & \multicolumn{5}{c}{ Conglomerados } \\
\cline { 2 - 6 } & 1 & 2 & 3 & 4 & 5 \\
\hline Graduados & 56,9 & 20,5 & 6,7 & 2,0 & 3,3 \\
$\mathrm{~N}$ & 28 & 91 & 24 & 85 & 38 \\
\hline
\end{tabular}

Fuente: Elaboración propia.

\subsubsection{Resultados}

La última dimensión considerada (Tabla 7) diferencia claramente los conglomerados 1 y 2 del resto de conjuntos: ambos tienen valores medios de acreditación de 6,1 y 5,4 años, respectivamente, más de tres años de lo que muestra el conglomerado que les sigue 
en años de acreditación promedio (conglomerado 5 con 2,5). Los conglomerados 3 y 4 tienen en promedio 0,2 y 1,4 años de acreditación, respectivamente.

Tabla 7

Media de variable de la dimensión resultados por conglomerado

\begin{tabular}{lccccc}
\hline \multirow{2}{*}{ Variable dimensión resultados } & \multicolumn{5}{c}{ Conglomerados } \\
\cline { 2 - 6 } & 1 & 2 & 3 & 4 & 5 \\
\hline Acreditación programa & 6,1 & 5,4 & 0,2 & 1,4 & 2,5 \\
$\mathrm{~N}$ & 28 & 91 & 24 & 85 & 38 \\
\hline
\end{tabular}

Fuente: Elaboración propia.

\subsection{Tipología de programas de doctorado en Chile}

A partir de la síntesis de los datos mostrados en el apartado anterior, se propone una tipología que consta de cinco categorías de programas de doctorado:

- Fuertes (conglomerado 1),

- Consolidados (conglomerado 2),

- Emergentes (conglomerado 5),

- En desarrollo (conglomerado 4) y

- Débiles (conglomerado 3).

En la Tabla 8 se muestran diferentes variables del sistema vinculadas con la tipología propuesta.

\subsubsection{Programas fuertes}

Son aquellos con mayor antigüedad del sistema, los que en promedio comenzaron sus actividades hace más de 20 años. El programa más joven de este conjunto tiene 11 años. El 70\% se desarrolla dentro de las áreas OCDE de Ciencias Naturales, Humanidades e Ingeniería y Tecnología. En su gran mayoría se implementan en universidades del CRUCH y, en términos de la taxonomía de Muñoz y Blanco (2013), más de un 70\% en universidades de investigación y un $25 \%$ en universidades de acreditación. Se concentran en la Región Metropolitana (75\%), poseen gran experiencia en procesos de 
acreditación y los mejores resultados del sistema respecto de los años que obtienen por parte de CNA: se concentran en los tramos medios y altos de acreditación (6-7 y 8-10 años).

Desde el punto de vista de sus estudiantes, son los programas que cuentan con mayor cantidad de matrícula total y de primer año, y los que tienen la menor edad promedio de todos los estudiantes matriculados en programas de doctorado del país. Presentan una proporción de estudiantes extranjeros del 10\% entre sus matriculados, lo que los sitúa ligeramente por debajo de la media del sistema (14\%). En relación con los graduados, los programas tradicionales casi cuadruplicaron el desempeño de la media del sistema durante el periodo 2007-2015: 57 graduados frente a 15, respectivamente.

Los programas fuertes han construido una sólida base de capacidades científicas como resultado de los recursos que han logrado captar a lo largo de sus trayectorias. Prácticamente todos han sido beneficiarios de financiamiento por parte del programa de Mecesup, algunos con proyectos desde 2000 hasta 2016, que han buscado —en etapas sucesivas - su fortalecimiento, consolidación, integración en redes e internacionalización. Por otro lado, al ser los programas con mayor antigüedad, fueron los destinatarios de los primeros esfuerzos sistemáticos del Estado para financiar becas de doctorado que, como se vio anteriormente, se concentraron en programas del área de ciencias naturales. En la actualidad, si bien estos programas corresponden al $11 \%$ de la oferta doctoral, captaron cerca del 30\% de los estudiantes del sistema y un 36\% del total de becas de doctorado nacional que Conicyt entregó en el periodo 20112016. Todos los que integran este grupo recibieron becas por parte de Conicyt en el periodo indicado.

Este tipo de programas, con gran capacidad para captar recursos de forma estable en el tiempo, se ha posicionado como referente nacional e internacional en las áreas disciplinarias en las que se desarrollan (ciencias biológicas y celulares, química, biotecnología, literatura, entre otras). Ejemplo de esto es que prácticamente la totalidad de este grupo fue considerado por el Mineduc como parte de los programas doctorales con mejor desempeño del país, destacando 
la productividad científica de sus claustros, estudiantes y egresados (Mineduc, 2014).

\subsubsection{Programas consolidados}

Constituyen poco más de un tercio de la oferta doctoral en el país, concentrando el $41 \%$ de la matrícula total y el $37 \%$ de la matrícula de primer año de 2016. Al igual que los fuertes, los programas consolidados se concentran preponderantemente en las áreas OCDE de Ciencias Naturales, Humanidades e Ingeniería y Tecnología. Un $42 \%$ de los doctorados en ciencias naturales y cerca del 50\% de los programas que se desarrollan en el ámbito de la ingeniería y tecnología se encuentran dentro de este conjunto. Programas de las áreas OCDE de Ciencias Sociales y Ciencias Agrícolas también integran este grupo, mostrando mayor diversidad que la encontrada dentro de los programas fuertes.

El 95\% de los programas consolidados se desarrolla en universidades del CRUCH, las que cuentan con acreditaciones desde cuatro y cinco años con el área electiva de investigación, esto es, universidades de investigación y de acreditación (Muñoz y Blanco, 2013). A pesar de que un 60\% se desarrolla en la Región Metropolitana, hay una participación relevante de otras regiones del país: Antofagasta, Valparaíso y Biobío, principalmente.

En términos de trayectoria, los programas consolidados comenzaron sus actividades como media en 1997, lo que también les ha permitido obtener experiencia en procesos de acreditación (tres procesos como media). En general, han tenido buenos resultados en materia de acreditación, con un desempeño ligeramente inferior al que muestran los programas fuertes, pero bastante superior al resto de programas ofertados en el sistema: más de dos años por sobre la media de acreditación del sistema (5,4 años frente a 3,3 años, respectivamente), concentrándose en los tramos medios de acreditación (4-5 y 6-7 años).

Desde el punto de vista de los recursos con los que cuentan, los programas consolidados poseen niveles de matrícula total y de primer año por sobre la media del sistema. Sus estudiantes tienen 
edades promedio ligeramente superiores a la de los estudiantes de los programas tradicionales, pero se encuentran dos años por debajo de la que presentan todos los matriculados en programas doctorales del país. En su composición, cerca de un quinto de sus matriculados son estudiantes extranjeros.

Estas características se vinculan con la capacidad de captar estudiantes con becas de doctorado de Conicyt, las que como media logran cubrir el $85 \%$ de la matrícula de primer año. En el periodo 2011-2016, todos los programas consolidados recibieron becas de este tipo, concentrando cerca del 50\% de las becas otorgadas por Conicyt. En relación con el financiamiento de tipo institucional, los programas consolidados han recibido apoyo estable y continuo del programa Mecesup: más del $80 \%$ ha sido beneficiario de este instrumento, con proyectos iniciados desde el año 2000.

En síntesis, las características típicas mencionadas de los programas consolidados muestran que este conjunto constituye una oferta doctoral con experiencia en procesos de aseguramiento de la calidad, capacidad de captar recursos para su desarrollo en el tiempo —en términos de estudiantes nacionales y extranjeros, becas y financiamiento-, que le permiten generar productos (graduados) y resultados (producción científica) que impactan en el sistema.

\subsubsection{Programas emergentes}

Este tipo de programas supone el 14\% de la oferta doctoral y el 9\% de la matrícula. Se desarrollan principalmente en universidades del $\mathrm{CRUCH}$, pero también en universidades privadas. Desde el punto de vista de la taxonomía de Muñoz y Blanco (2013), hay presencia de universidades elitistas, masivas y no elitistas, que se suman a las ya mencionadas de investigación y acreditación. En términos de la acreditación de las universidades que albergan estos programas, todas tienen desde cuatro años en adelante, salvo en un caso, que se desarrolla en una universidad no acreditada.

Además de las áreas OCDE habituales de desarrollo, dentro de los programas emergentes destacan aquellos del área de Ciencias Sociales. Desde la óptica territorial, estos programas se desenvuelven 
en las regiones del Biobío y Valparaíso; la Región Metropolitana tiene uno de los menores pesos relativos en comparación con los otros tipos. Aparecen también de forma incipiente otras regiones del país: Arica y Parinacota, Atacama, Coquimbo y Los Ríos.

En relación con su trayectoria y experiencia, estos programas poseen como media seis a siete años de antigüedad y cerca de dos procesos de acreditación con CNA. Los resultados de estos procesos no son tan exitosos como los que presentan los programas fuertes y consolidados, ya que se sitúan ligeramente por debajo de la media del sistema. Se concentran en niveles medios y bajos de acreditación: tramos 2-3 y 4-5 años. Sin embargo, también se presentan programas no acreditados y fuera de sistema. Este tipo de programas posee la mayor diversidad en relación con los niveles de acreditación, lo que permite conjeturar que esta categoría de programas se caracteriza por su dinamismo, aunando trayectorias ascendentes y descendentes en el sistema.

Respecto de la cantidad y calidad de recursos que son capaces de atraer, hay que destacar los siguientes elementos: una matrícula total y de primer año por debajo de la media del sistema; una edad promedio de sus matriculados de 34 años, cerca de tres años superior a la que presentan los estudiantes de los programas fuertes; un $42 \%$ de sus matriculados son estudiantes extranjeros, lo que constituye la mayor proporción del sistema y una característica clave de este tipo de programas. En relación con las becas de doctorado, si bien más del 70\% del conjunto ha obtenido este beneficio por parte de Conicyt en el periodo 2011-2016, en términos generales la cobertura alcanza a cuatro de cada 10 estudiantes de primer año. Además, menos de la mitad del conjunto ha recibido financiamiento por parte del programa Mecesup, fundamentalmente para apoyar etapas de diseño y desarrollo temprano de los programas.

La importante proporción de estudiantes extranjeros sugiere en estos programas la presencia de una estrategia de diversificación de sus fuentes de financiamiento, con el objetivo de enfrentar la escasez de recursos provenientes del sector público. Este es el elemento distintivo del conjunto de programas emergentes. 


\subsubsection{Programas en desarrollo}

Un tercio de la oferta doctoral en el país se puede caracterizar como en desarrollo, la que concentra el 15\% de la matrícula. Uno de cada cuatro de estos programas es implementado en universidades privadas, 15\% del tipo elitista (Muñoz y Blanco, 2013). Desde el punto de vista territorial, están presentes en las 12 regiones del país que albergan programas de doctorado y se destacan, además de la Metropolitana, las regiones de Valparaíso, Maule, Biobío y La Araucanía. Este tipo de programas se desarrolla en su totalidad en instituciones acreditadas y se concentran preferentemente en el tramo de 4-5 años, más el área electiva de investigación. Los programas en desarrollo presentan la mayor diversidad en términos institucionales y regionales, combinando diferentes proyectos educativos, tamaños, orientaciones, tradiciones y entornos socioeconómicos.

Los programas en desarrollo muestran el mayor equilibrio disciplinar entre las cinco tipologías: las áreas OCDE de Ciencias Naturales y Ciencias Sociales predominan (con proporciones cercanas al 23\%); las áreas de Ingeniería y Tecnología, Ciencias Médicas y de Salud y Humanidades, tienen pesos similares (alrededor del 12-14\%); y las áreas de Ciencias Agrícolas y Educación tienen una participación del 8\% aproximadamente. Esta distribución equilibrada entre áreas, sumada a la característica antes mencionada de diversidad institucional de las universidades que los albergan, remarca el elemento distintivo de este tipo de programas: el desarrollo de proyectos académicos con orientación hacia la investigación dentro de diferentes entornos socioeducativos. Entre estos programas hay también un énfasis hacia lo multidisciplinar y colaborativo. No es casual que estos programas sean los más jóvenes del sistema, con una antigüedad media de 5-6 años.

Los programas en desarrollo presentan niveles de matrícula total y de primer año entre un 50\% y 35\% por debajo de la media del sistema, respectivamente. Sin embargo, han logrado atraer a estudiantes con promedios de edad inferiores a los programas emergentes. En términos de becas de doctorado otorgadas por Conicyt, las que se encuentran condicionadas a la acreditación de los programas, los programas en desarrollo han conseguido en 
general una cobertura del $25 \%$ de su matrícula de primer año, y el $44 \%$ de estos programas ha accedido a becas en el periodo de referencia (2011-2016). Este carácter en desarrollo se subraya por el hecho de que los pocos que recibieron financiamiento por parte de Mecesup (21\% del conjunto), lo hicieron con el objeto de diseñar sus programas o al alero de proyectos de fortalecimiento institucional en áreas científicas más amplias.

Desde el punto de vista de su experiencia en procesos de acreditación, los programas en desarrollo poseen una media de un proceso con CNA (con 1,4 años de acreditación promedio) y se sitúan en los tramos inferiores: no acreditado y 2-3 años. Más de un tercio de ellos, al momento del análisis de los datos, no se había presentado a procesos de acreditación. Estos programas poseen debilidades importantes en relación con la calidad de la enseñanza que entregan: la mitad se encuentra acreditado en el tramo más bajo y la otra mitad no está acreditado, o bien, no se ha sometido a una mirada externa por parte de CNA. En este sentido, poseen un nivel de riesgo e incertidumbre asociados, pudiéndose presentar también trayectorias descendentes de calidad.

\subsubsection{Programas débiles}

Corresponde al $9 \%$ de la oferta y concentra el 6\% de la matrícula del país. En términos relativos, las universidades privadas tienen aquí su mayor peso (33\%) y el restante $67 \%$ se desarrolla en universidades estatales. Universidades de acreditación y no elitistas (Muñoz y Blanco, 2013) concentran cerca del 90\% de este tipo de programas. Dos tercios de este conjunto se desarrolla en la Región Metropolitana. En términos de acreditación institucional, los programas débiles son implementados en universidades ubicadas en todos los tramos: desde las no acreditadas hasta las acreditadas por siete años.

Más del 50\% de estos programas pertenecen a las áreas OCDE de Ciencias Sociales y Educación, las más tardías en desarrollarse en el país. La mayoría de estos programas no tiene experiencias en procesos de acreditación, y aquellos que se han sometido a una mirada externa por parte de CNA, han resultado no acreditados. 
A pesar de que el $75 \%$ de estos programas se implementan en universidades acreditadas, solo un $8 \%$ ha logrado un nivel mínimo de calidad certificado por CNA. Su antigüedad promedio es de más de nueve años, esto es mayor a la de los programas emergentes y en desarrollo, lo que nos indica que las trayectorias de calidad no son lineales ni ascendentes.

Esta divergencia entre acreditación institucional y acreditación de programas del nivel doctoral señala una de las características del sistema chileno: inexistencia de regulación en el origen de los programas, quedando su implementación y desarrollo sujetos a las decisiones autónomas y contingentes de universidades y departamentos implicados.

Los programas débiles, a pesar de que no poseen los niveles de calidad esperados de este nivel de enseñanza, han logrado captar estudiantes. Sus indicadores de matrícula total y de primer año son superiores a los de programas emergentes y en desarrollo. Presentan, como media, un 5\% de matriculados extranjeros. Sus niveles de generación de graduados en el periodo 2007-2015 son mayores que los que presentan los programas emergentes y en desarrollo. La característica más notable de estos programas respecto de los estudiantes que atraen, es que tienen 46 años promedio de edad, esto es, cerca de 12 años sobre la media del sistema. Los datos sugieren que estos programas funcionan como un vehículo para consolidar posiciones profesionales, más que para el desarrollo de trayectorias de investigación al más alto nivel. 
220 PROGRAMAS DOCTORALES EN CHILE: UNA PROPUESTA TIPOLÓGICA PARA INTERPRETAR SU DIVERSIDAD - P. Baeza

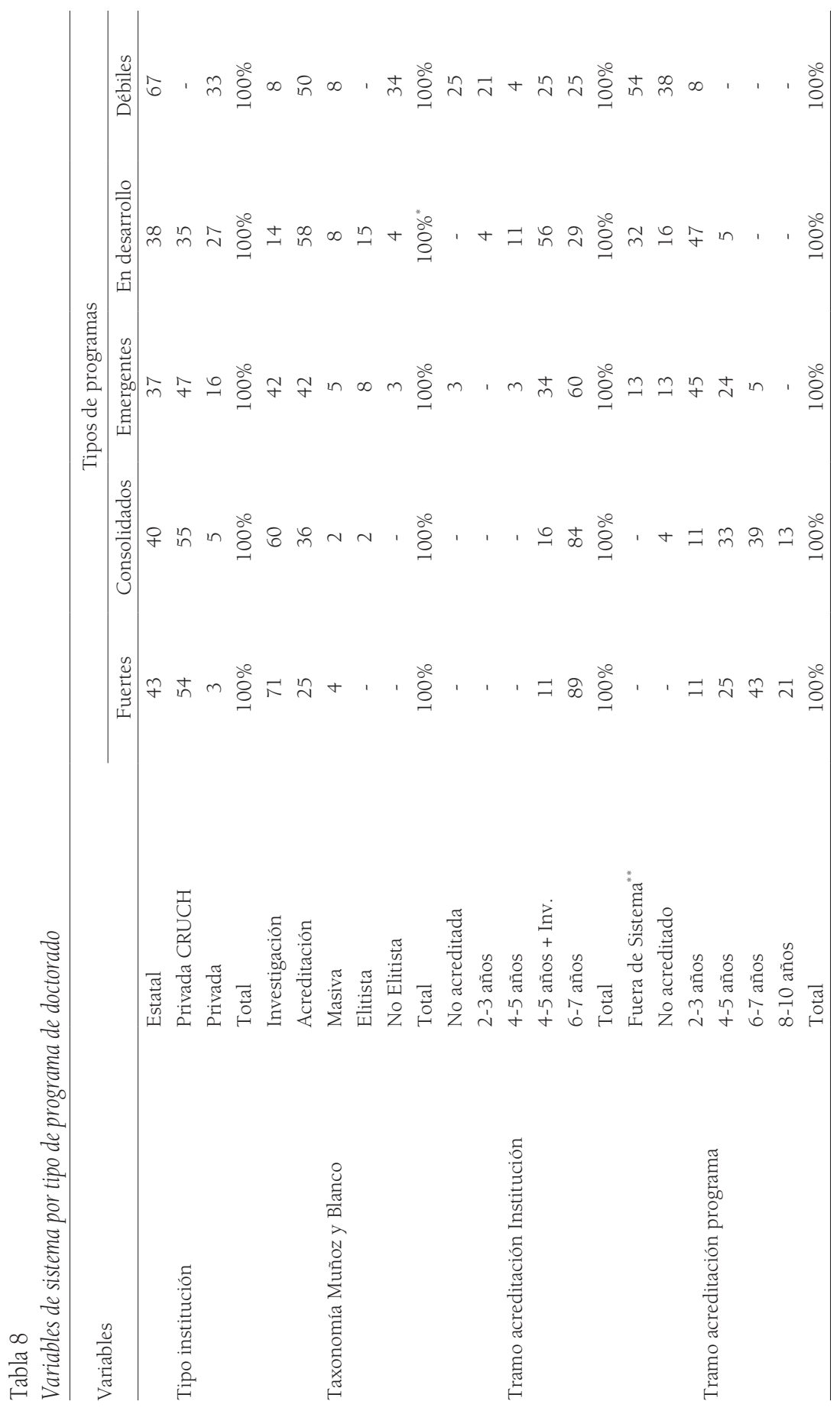




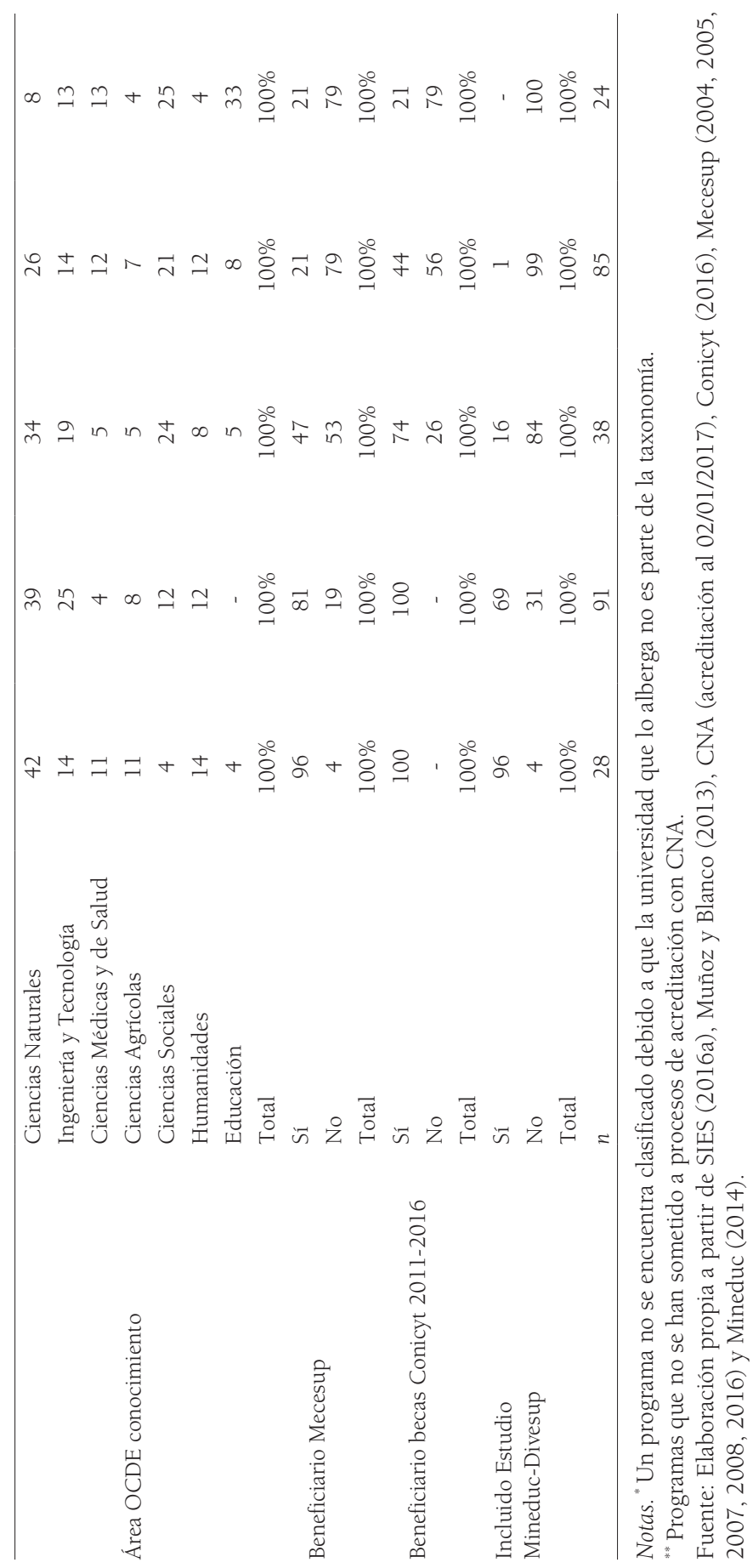




\section{Conclusiones}

El presente trabajo es un esfuerzo sistemático por comprender la diversidad de la oferta doctoral en Chile. La contribución de este estudio es doble. En primer lugar, la estrategia metodológica utilizada - análisis de conglomerados como base para una construcción tipológica - contribuye a este objetivo desde una práctica circular: desde lo teórico a lo empírico y desde lo empírico a lo teórico. Esto nos permite superar posiciones reduccionistas basadas en lo conceptual —donde los datos son utilizados solamente para ejemplificar las definiciones categoriales- o en lo empírico, lo que supone que los datos hablan por sí mismos.

En segundo lugar, la tipología propuesta es una herramienta interpretativa de las diferentes realidades de los programas de doctorado desarrollados en Chile, que captura sus elementos típicos y los agrupa en conjuntos homogéneos, aportando al entendimiento de la diversidad horizontal dentro de la oferta (Ziegele, 2013). Dado el propio dinamismo del desarrollo de los programas, la tipología incorpora los aspectos más relevantes que los caracterizan para enfocarse en una mirada comprehensiva del sistema y no detenerse en las particularidades de uno u otro programa. No pretende jerarquizarlos, ordenándolos bajo una lógica atributiva, es decir, de poseer o no determinados elementos (estudiantes, financiamiento, graduados), sino comprender, bajo una lógica relacional, las condiciones que les permiten situarse en posiciones más o menos ventajosas en el sistema. En este sentido, este trabajo busca contribuir al desarrollo de investigación — sustentada en información cuantitativa y cualitativa - que permita interpretar la cambiante realidad de la educación de posgrado en Chile.

Una de las limitaciones con las que ha tenido que lidiar este estudio es la escasez de datos respecto de los programas de doctorado. El sistema no cuenta con información sistemática acerca de las características de los estudiantes y académicos de los claustros (experiencia, productividad científica), recursos financieros de los programas (becas, fondos de investigación), recursos de infraestructura y equipamiento, redes de investigación con las que 
se vinculan, progresión académica de los estudiantes (retención, graduación), inserción laboral de sus egresados, contribución e impacto en la disciplina en la que se enmarcan, solo por mencionar algunas dimensiones relevantes. Chile debe contar no solo con un sistema de información integrado, robusto y público, sino también con prácticas de transparencia activa de los programas acerca de sus características, procesos académicos, resultados e impacto. Este es un déficit que es necesario corregir si se quiere introducir mayor transparencia en el sistema de educación superior del país.

Los resultados del estudio muestran la diversidad existente en la oferta doctoral en el país. Considerando la tipología como un continuum (fuertes-consolidados-emergentes-en desarrollo-débiles), se aprecia la gran diferencia entre ambos extremos: los programas fuertes y consolidados —que concentran el 45\% de la oferta, el 70\% de la matrícula total y el 88\% de los graduados del periodo 2007-2015poseen antigüedad, experiencia en procesos de acreditación y niveles de calidad muy superiores a los programas en desarrollo y débiles (que concentran $41 \%$ de la oferta, $21 \%$ de matrícula y $8 \%$ de graduados).

El primer subconjunto (programas fuertes/consolidados) se desarrolla en universidades de investigación y acreditación (Muñoz y Blanco, 2013), fuertemente concentrados en la Región Metropolitana, de Valparaíso y del Biobío, y contaron con financiamiento sostenido de Mecesup y becas de Conicyt. El segundo subconjunto (programas en desarrollo/débiles) muestra una importante participación de universidades masivas, elitistas y no elitistas (Muñoz y Blanco, 2013), y se desarrollan a lo largo de todo el territorio, pero con escaso financiamiento público.

En una posición de bisagra en el continuum se ubican los programas emergentes (14\% de la oferta y $9 \%$ de la matrícula). Este grupo se desarrolla principalmente en universidades de investigación y acreditación de las regiones de Valparaíso y Biobío, ha contado con financiamiento público para su desarrollo y posee la mayor proporción de estudiantes extranjeros dentro de su matrícula (42\%). Si bien contar con una gran cantidad de estudiantes internacionales no significa necesariamente la internacionalización de los programas 
(de Wit, 2011), sí indica al menos dos elementos: capacidad de los programas por captar recursos dentro de este nuevo mercado global (Findlay, McCollum \& Packwood, 2017); y la posibilidad de generar redes de investigación internacionales e incorporar diferentes visiones culturales en la enseñanza, lo que contribuye a ampliar sus alcances (Nerad, 2010).

La diversidad, considerada como una cualidad positiva en el campo de la educación superior, al posibilitar la inclusión en el sistema de un mayor número de estudiantes — con trayectorias y necesidades diferenciadas, además de satisfacer las demandas de los nuevos y complejos requerimientos de la sociedad del conocimiento (Birnbaum, 1983; Huisman, 1995; Meek et al., 2000; Scott, 2015; Trow, 2000; van Vught, 2007, 2008)— se muestra, para el caso de la educación doctoral en Chile, como una característica ambivalente: de un lado, se observa el desarrollo de programas doctorales en nuevas áreas del conocimiento dentro de contextos territoriales diferentes al de la capital y con nuevas características (multidisciplinares, programas en consorcio, vinculados a realidades territoriales, entre otras); y por otro, las diferencias en términos de recursos, procesos, productos y resultados entre los cinco tipos de programas, muestran que hoy el sistema no asegura niveles de calidad equivalentes para los estudiantes de doctorado.

Desde una perspectiva de aseguramiento de la calidad de la educación doctoral en Chile, la nueva ley de Educación Superior (Ley $\left.\mathrm{N}^{\circ} 21.091,2018\right)$ supone una oportunidad para modificar el diseño de la acreditación de programas en este nivel. Al consagrar dicha ley la acreditación obligatoria de los programas doctorales se dan dos señales fuertes al sistema, como son: 1) transparentar la oferta presente en el país, ofreciendo garantía pública de su calidad a la comunidad; 2) consolidar el nivel doctoral como clave en el desarrollo de las capacidades científicas del país, el que todavía tiene déficits importantes en cuanto al número de investigadores en relación con su población activa (Barro, Fernández $\&$ Santelices, 2015).

Algunos elementos derivados de los resultados de esta investigación se pueden considerar en este nuevo escenario. En 
primer lugar, la diversidad en el sistema obliga a explorar mecanismos diferenciados de aseguramiento de la calidad en relación con los "ciclos de vida" de los programas. Programas con experiencia y buenos resultados — con al menos dos procesos de acreditación y en tramos superiores respecto de los años de acreditación- no deben ser sometidos a idénticos procesos que programas nuevos o con manifiestas debilidades. Los primeros deben ser parte de un seguimiento periódico a través de indicadores clave (matrícula, graduados, productividad científica del claustro, estudiantes y egresados, inserción de egresados, equipamiento, vinculación con el medio, entre otros), y ser apoyados para alcanzar niveles de calidad internacionales. Respecto de los segundos, el sistema debiese comenzar a evaluarlos incluso antes de que comiencen sus actividades, como proyectos educativos, de forma de garantizar umbrales de calidad mínimos para los estudiantes. Para estos programas, el énfasis debiese estar puesto en el acompañamiento de su proceso de desarrollo.

En segundo lugar, y dadas las asimetrías geográficas y entre áreas del conocimiento, se deben considerar otras prácticas para impactar positivamente en el mejoramiento de la calidad de la oferta. Incentivar los programas mediante el diseño de indicadores de colaboración, para que se vinculen entre sí a través de la movilidad de académicos y estudiantes, uso de equipamiento e instalaciones, producción científica conjunta, entre otros. Este incentivo podría generar dinámicas virtuosas para el sistema, mejorando indicadores de resultados globales (como publicaciones indexadas desarrolladas en colaboración) y particulares, impulsando el perfeccionamiento de programas en desarrollo.

Un nuevo modelo de acreditación de los programas de doctorado debe conservar los elementos ya asumidos por el sistema —consistencia interna del diseño (objetivo, carácter, estructura curricular, plan de estudios, entre otros), existencia de claustros académicos sólidos, sustentabilidad de las líneas de investigación, foco en los resultados y productividad-e incorporar aquellos que apunten a fomentar la colaboración y asociatividad, pertinencia de los proyectos formativos, orientación hacia la excelencia e 
internacionalización —esto último fundamentalmente en programas fuertes y consolidados-. Esta investigación pretende contribuir con evidencia al análisis y desarrollo de esta línea de desarrollo clave para el país.

\section{Referencias}

Altbach, P. G. (2013). The international imperative in higher education. Global perspectives on Higher Education, Vol 27. Rotterdam: Sense Publishers.

Altbach, P. G., Reisberg, L., \& Rumbley, L. E. (2009). Trends in global higher education: Tracking an academic revolution. A report prepared for the Unesco 2009 World Conference on Higher Education. Paris: Unesco.

Aksnes, D. W., Rørstad, K., Piro, F., \& Sivertsen, G. (2011). Are female researchers less cited? A large-scale study of Norwegian scientists. Journal of the American Society for Information Science and Technology, 62(4), 628-636. https://doi.org/10.1002/asi.21486

Baeza, P. (2017). Diversidad y diferenciación en la oferta de programas de doctorado en Chile. Calidad en la Educación, 47, 179-214. https://doi.org/10.31619/caledu.n47.34

Bailey, K. D. (1994). Quantitative applications in the social sciences: Typologies and taxonomies. Thousand Oaks, CA: SAGE Publications

Barjak, F. (2006). Research productivity in the internet era. Scientometrics, 68(3), 343-360. https://doi.org/10.1007/s11192-006-0116-y

Barro, S., Fernández, S., y Santelices, B. (2015). Los sistemas iberoamericanos de ciencia e innovación en el arranque del siglo XXI. En S. Barro (Coord.), La transferencia de I+D, la innovación y el emprendimiento en las universidades. Educación superior en Iberoamérica - Informe 2015 (pp. 51-82). Santiago de Chile: RIL editores.

Birnbaum, R. (1983). Maintaining diversity in higher education. San Francisco: The Jossey-Bass Higher Education Series.

Boosten, K., Vandevelde, K., Derycke, H., te Kaat, A., \& Van Rossem, R. (2014). Careers of Doctorate Holders Survey 2010. RED and innovation in Belgium Research Series, 13. Recuperado de https://www.belspo.be/ belspo/scienceconnection/012/careersdoctorateholders.pdf

Brint, S., Riddle, M., \& Hanneman, R. (2006). Reference sets, identities, and aspirations in a complex organizational field: The case of American four-year colleges and universities. Sociology of Education, 79(3), 229252. https://doi.org/10.1177/003804070607900303 
Brunner, J. J. (1986). Informe sobre la educación superior en Chile. Santiago de Chile: Flacso.

Brunner, J. J. (2009). Tipología y características de las universidades chilenas. Documento para comentarios. Recuperado de http://200.6.99.248/ bru487cl/files/Tipol\%26Caract_080209.pdf

Brunner, J. J. (2013). Sobre la clasificación de universidades. En M. V. Santelices, J. J. Ugarte, y J. Salmi (Eds.), Clasificación de instituciones de educación superior (pp. 109-134). Santiago de Chile: Ministerio de Educación.

Brunner, J. J., Elacqua, G., Tillett, A., Bonnefoy, J., González, S., Pacheco, P., y Salazar, F. (2005). Guiar el mercado. Informe sobre la educación superior en Chile. Santiago de Chile: Universidad Adolfo Ibáñez.

Cea D’Ancona, M. Á. (2004). Análisis multivariable. Teoría y práctica en la investigación social. Madrid: Editorial Síntesis.

Celis, S. y Véliz, D. (2017). La acreditación como agente de mejora continua en los programas de postgrado en Ciencia y Tecnología, Vol. 5. Santiago de Chile: Comisión Nacional de Acreditación. Serie Cuadernos de Investigación en Aseguramiento de la Calidad.

Centro Interuniversitario de Desarrollo, Cinda. (2012). Aseguramiento de la calidad en Iberoamérica. Educación Superior. Informe 2012. Santiago de Chile: Autor.

Cheol Shin, J. (2009). Classifying higher education institutions in Korea: A performance-based approach. Higher Education, 57(2), 247-266. https://doi.org/10.1007/s10734-008-9150-4

Codling, A. \& Meek, L. (2006). Twelve propositions on diversity in higher education. Higher Education Management and Policy, 18(3), 1-24. https://doi.org/10.1787/hemp-v18-art17-en

Comisión Nacional de Acreditación, CNA. (2016). Operacionalización de criterios de evaluación para la acreditación de programas de postgrado: doctorado, magíster académico y magister profesional. Recuperado de https://www.cnachile.cl/Documentos\%20de\%20Paginas/ Operacionalizaci\%c3\%b3n\%20de\%20criterios\%20de\%20Postgrado. pdf

Comisión Nacional de Acreditación, CNA. (2017). Buscador avanzado de acreditaciones. Recuperado de www.cnachile.cl

Comisión Nacional de Ciencia y Tecnología, Conicyt. (2016). Becas de doctorado nacional. Recuperado de http://www.Conicyt.cl/becasConicyt/estadisticas/becarios-nacionales/doctorado-nacional/ 
Consejo Nacional de Educación, CNED. (2016). INDICES Matrícula pregrado-postgrado, años 2005-2016. Recuperado de http://www. cned.cl/bases-de-datos

de Wit, H. (2011). Internationalization of higher education: Nine misconceptions. International Higher Education, 64, 6-7. https://doi.org/10.6017/ihe.2011.64.8556

Devés, R. y Marshall, M. T. (2008). El desarrollo del posgrado en Chile. En J. J. Brunner y C. Peña (Eds.), Reforma a la educación superior (pp. 265-304). Santiago de Chile: Ediciones Universidad Diego Portales.

Didriksson, A. (2012). La nueva agenda de transformación de la educación superior en América Latina. Perfiles Educativos, 34(138), 184-203.

Diem, A. \& Wolter, S. C. (2013). The use of bibliometrics to measure research performance in education sciences. Research in Higher Education, 54(1), 86-114. https://doi.org/10.1007/s11162-012-9264-5

Dooner, C., Armanet, L., Busco, C., d'Alencon, A., y Salomone, A. (2016). Impacto de los procesos de autoevaluación en la gestión de pregrado y postgrado en la Universidad de Chile (2011-2014). Santiago de Chile: Comisión Nacional de Acreditación. Serie Cuadernos de Investigación en Aseguramiento de la Calidad.

Everitt, B., Landau, S., Leese, M., \& Stahl, D. (2011). Cluster Analysis. London: Wiley.

Findlay, A., McCollum, D., \& Packwood, H. (2017). Marketization, marketing and the production of international student migration. International Migration, 55(3), 139-155. https://doi.org/10.1111/imig.12330

Gingras, Y., Larivière, V., Macaluso, B., \& Robitaille, J. P. (2008). The effects of aging on researchers' publication and citation patterns. PLoS One, 3(12), e4048 https://doi.org/10.1371/journal.pone.0004048

Goedegebuure, L. \& Meek, L. (1997). On change and diversity: The role of governmental policy and environmental influences. Higher Education in Europe, 22(3), 309-319. https://doi.org/10.1080/0379772970220305

Harvey, L. \& Green, D. (1993). Defining quality. Assessment \& Evaluation in Higher Education, 18(1), 9-34.

Huberty, C., Jordan, M., \& Brandt, C. (2005). Cluster analysis in Higher Education research. En J. Smart (Ed.), Higher education: Handbook of theory and research, 20 (pp. 437-457). London: Springer.

Huisman, J. (1995). Differentiation, diversity and dependency in higher education. A theoretical and empirical analysis. Utrecht: Lemma. 
Huisman, J. (2000). Higher education institutions: as different as chalk and cheese? Higher Education Policy, 13, 41-53.

https://doi.org/10.1057/palgrave.hep.8390138

Huisman, J., Meek, L., \& Wood, F. (2007). Institutional diversity in higher education: A cross-national and longitudinal analysis. Higher Education Quarterly, 61(4), 563-577.

https://doi.org/10.1111/j.1468-2273.2007.00372.x

Kells, H. R. (1992). Performance indicators for higher education: A critical review with policy recommendations. Population and Human Resources Department. Education and Employment Division background (Paper series; no PHREE 92/56). Washington, D.C.: The World Bank. Recuperado de http://documents.worldbank.org/curated/en/430761468766509535/ Performance-indicators-for-higher-education-a-critical-review-withpolicy-recommendations

Ley N 21.091 Sobre Educación Superior del Ministerio de Educación. Diario Oficial de la República de Chile, Santiago, Chile, 29 de mayo 2018.

Martin, M. \& Stella, A. (2007). External quality assurance in higher education: Making choices. Paris: United Nations.

McCormick, A. (2013). Classifying higher education institutions: Lessons from the Carnegie classification. Pensamiento Educativo. Revista de Investigación Educacional Latinoamericana, 50(1), 65-75.

https://doi.org/10.7764/pel.50.1.2013.6

Mejoramiento de la Calidad y la Equidad en la Educación Superior, Mecesup (2004). Mecesup. Memoria de proyectos 1999, Fondo Competitivo. Santiago de Chile: Ministerio de Educación.

Mejoramiento de la Calidad y la Equidad en la Educación Superior, Mecesup (2005). Mecesup. Memoria de proyectos 2000, Fondo Competitivo. Santiago de Chile: Ministerio de Educación.

Mejoramiento de la Calidad y la Equidad en la Educación Superior, Mecesup (2007). Mecesup. Memoria de proyectos 2001-2002. Santiago de Chile: Ministerio de Educación.

Mejoramiento de la Calidad y la Equidad en la Educación Superior, Mecesup (2008). Mecesup. Memoria de proyectos 2003, Fondo Competitivo. Santiago de Chile: Ministerio de Educación.

Mejoramiento de la Calidad y la Equidad en la Educación Superior, Mecesup (2016). Apoyo programas de doctorado. Proyectos 1999-2014. Santiago de Chile: Ministerio de Educación. Obtenido de Comunicación personal del Dr. Ricardo Reich. 
Meek, L., Goedegebuure, 1., \& Huisman, J. (2000). Understanding diversity and differentiation in higher education: an overview. Journal of Higher Education Policy, 13, 1-6. https://doi.org/10.1057/palgrave.hep.8390141

Merton, R. K. (1968). The Matthew effect in science. Science, 159(3810), 5663. https://doi.org/10.1126/science.159.3810.56

Merton, R. K. \& Zuckerman, H. (1973). Age, aging and age structure in science. En R. K. Merton (Ed.), The sociology of science (pp. 497-556). Chicago: University of Chicago Press.

Milligan, G. \& Cooper, M. (1985). An examination of procedures for determining the number of clusters in dataset. Psychometrika, 50(2), 159-179. https://doi.org/10.1007/bf02294245

Milligan, G. \& Cooper, M. (1987). Methodology review: Clustering methods. Applied Psychological Measurement, 11(4), 329-354. https://doi.org/10.1177/014662168701100401

Ministerio de Educación de Chile, Mineduc. (2014). Estudio evaluativo de un conjunto de programas doctorales chilenos 2013-2014. Santiago de Chile: Autor.

Munita, M. I. y Reyes, J. (2012). El sistema de postgrado en Chile: evolución y proyecciones para las universidades del Consejo de Rectores. Santiago de Chile: Editorial Universidad de Valparaíso.

Muñoz, M. y Blanco, C. (2013). Una taxonomía de las universidades chilenas. Calidad en la Educación, 38, 181-213. https://doi.org/10.31619/caledu.n38.109

Nerad, M. (2010). Globalization and the internationalization of graduate education: A macro and micro view. Canadian Journal of Higher Education, 40(1), 1-12.

Parada, J. R. (2010). Universidades públicas y privadas: un enfoque tridimensional. Estudios Públicos, 120, 183-205. Recuperado de https:// www.cepchile.cl/cep/site/artic/20160304/asocfile/20160304095425/ rev120_JRParada.pdf

Pusser, B. \& Marginson, S. (2013). University rankings in critical perspective. The Journal of Higher Education, 84(4), 544-568. https://doi.org/10.1353/jhe.2013.0022

Rama, C. (2015). Los sistemas iberoamericanos de educación superior. Investigación y diversificación. En S. Barro (Coord.), La transferencia de I+D, la innovación y el emprendimiento en las universidades. Educación superior en Iberoamérica-Informe 2015 (pp. 95-118). Santiago de Chile: RIL editores. 
Reyes, C. y Rosso, P. P. (2013). Una nueva clasificación de las universidades chilenas. En M. V. Santelices, J. J. Ugarte y J. Salmi (Eds.), Clasificación de instituciones de educación superior (pp. 135-151). Santiago de Chile: Ministerio de Educación.

Rhoades, G. \& Slaughter, S. (1997). Academic capitalism, managed professionals, and supply-side higher education. Social Text, 51, 9-38. https://doi.org/10.2307/466645

Rhoades, G. \& Slaughter, S. (2004). Academic capitalism in the new economy: Challenges and choices. American Academic, 1(1), 37-59.

Rørstad, K. \& Aksnes, D. W. (2015). Publication rate expressed by age, gender and academic position - A large-scale analysis of Norwegian academic staff. Journal of Informetrics, 9(2), 317-333. https://doi.org/10.1016/j.joi.2015.02.003

Rousseeuw, P. (1987). Silhouettes: A graphical aid to the interpretation and validation of cluster analysis. Journal of Computational and Applied Mathematics, 20, 53-65. https://doi.org/10.1016/0377-0427(87)90125-7

Sabharwal, M. (2013). Comparing research productivity across disciplines and career stages. Journal of Comparative Policy Analysis, 15(2), 141-163. https://doi.org/10.1080/13876988.2013.785149

Scott, P. (2015). Markets and managerialism. Enhancing diversity or promoting conformity? En R. Pritchard, M. Klumpp, \& U. Teichler (Eds.), Diversity and excellence in higher education. Can the challenges be reconciled? (pp. 3-17). Rotterdam: Sense Publishers.

Servicio de Información de Educación Superior, SIES. (2015). Compendio histórico de educación superior. Recuperado de http://www.mifuturo. cl/index.php/estudios/estructura-compendio

Servicio de Información de Educación Superior, SIES. (2016a). Matrícula 2016. Recuperado de http://www.mifuturo.cl/index.php/informes-sies/ matriculados

Servicio de Información de Educación Superior, SIES. (2016b). Titulados 2007-2015. Recuperado de http://www.mifuturo.cl/index.php/basesde-datos/titulados

Slaughter, S. \& Leslie, L. (2001). Expanding and elaborating the concept of academic capitalism. Organization, 8(2), 154-161. http://dx.doi.org/10.1177/1350508401082003

StataCorp. (2015). Stata multivariate statistics. Reference manual. Release 14. Texas: Stata Press Publication. 
Stensaker, B. (2007). Quality as fashion: Exploring the translation of a management idea into higher education. En D. F. Westerheijden, B. Stensaker, \& M. J. Rosa (Eds.), Quality assurance in higher education: Trends in regulation, translation and transformation (pp. 99-118). Dordrecht: Springer.

Stirling, A. (2007). A general framework for analyzing diversity in science, technology and society. Journal of the Royal Society Interface, 4, 707719. https://doi.org/10.1098/rsif.2007.0213

Torres, R. y Zenteno, M. E. (2011). El sistema de educación superior. Una mirada a las instituciones y sus características. En M. Jiménez de la Jara y F. Lagos (Eds.), Nueva geografía de la educación superior y de los estudiantes. Una cartografía del sistema chileno, su actual alumnado y sus principales tendencias (pp. 13-72). Santiago de Chile: Ediciones Universidad San Sebastián.

Trow, M. (2007). Reflections on the transition from elite to mass to universal access: Forms and phases of higher education in modern societies since WWII. En J. Forest \& P. Altbach (Eds.), International handbook of higher education, Vol. 18, (pp. 243-280). Dordrecht: Springer.

Trow, M. (2000). From mass Higher Education to universal access. The American advantage. Research and occasional (Paper Series, CSHE, 1.00). Recuperado de https://cshe.berkeley.edu/sites/default/files/ publications/pp.trow.masshe.1.00.pdf

van der Wende, M. (2008). Rankings and classifications in higher education: A European perspective. En J. C. Smart (Ed.), Higher education: Handbook of theory and research (pp. 49-71). Amsterdam: Springer.

van Vught, F. (2007). Diversity and differentiation in higher education systems. CHET Anniversary Conference. Cape Town: CHET.

van Vught, F. (2008). Mission diversity and reputation in higher education. Higher Education Policy, 21, 151-174. https://doi.org/10.1057/hep.2008.5

van Vught, F., Balanskat, A., Benneworth, P., Botas, P., Brandenburg, U., Burquel, N., ... Mora, J. (2010a). A university benchmarking handbook. Benchmarking in uropean Higher Education. Brussels: ESMU.

van Vught, F. A., Kaiser, F., Flle, J. M., Gaethgens, C., Peter, R., \& Westerheijden, D. F. (2010b). The European classification of higher education institutions. Enschede: Center for Higher Education Policy Studies (CHEPS).

van Vught, F., Bartelse, J., Bohmert, D., Burquel, N., Divis, J., Huisman, J., \& van der Wende, M. (2005). Institutional profiles. Towards 
a typology of higher education institutions in Europe. Report to the European Commission. Recuperado de http://doc.utwente.nl/53776/1/ engreport05institutionalprofiles.pdf

Williamson, C. (2012). Universidad y lucro. Recuperado de https://www. cepchile.cl/cep/site/artic/20160304/asocfile/20160304100152/ rev128_CWilliamson.pdf

Zapata, G. y Tejeda, I. (2009). Impactos del aseguramiento de la calidad y acreditación de la educación superior. Consideraciones y proposiciones. Calidad en la Educación, 31, 191-209.

https://doi.org/10.31619/caledu.n31.168

Ziegele, F. (2013). Classification of higher education institutions: The European case. Pensamiento Educativo. Revista de Investigación Educacional Latinoamericana, 50(1), 76-95.

https://doi.org/10.7764/pel.50.1.2013.7

Recibido: 22/01/2018

Aceptado 23/08/2018 